\title{
PASADO Y PRESENTE DE UN CENTRO PÚBLICO INTEGRADO DE GALICIA
}

\section{PAST AND PRESENT OF AN INTEGRATED PUBLIC CENTER OF GALICIA}

http://dx.doi.org/10.15304/ie.28.5514

\author{
Ana $M^{\mathbf{a}}$ Porto Castro \\ Universidad de Santiago de Compostela \\ anamaria.porto@usc.es
}

Isabel Sánchez Manso

Profesora de Educación Secundaria (jubilada)

ismanso@edu.xunta.es

\section{RESUMEN}

La Ley Orgánica 1/1990 prevé el funcionamiento de centros integrados que abarcan dos o más niveles de enseñanza y el Real Decreto 1004/1991, de 14 de junio, en su Disposición adicional primera considera el funcionamiento de centros de diferentes enseñanzas en el mismo edificio o recinto escolar, determinando los espacios que son comunes y posibilitando formas organizativas más flexibles para adaptarse a las distintas situaciones que la realidad escolar puede presentar. En este marco, la Consellería de Educación e Ordenación Universitaria implanta y regula la existencia en la organización educativa de la Comunidad Autónoma de Galicia de los centros públicos integrados, CPI (Decreto 7/1999, de 7 de enero, DOG núm.16, martes 26 de enero de 1999).

Reflexionar sobre estas cuestiones, las oportunidades que se derivan de la puesta en marcha de los centros públicos integrados para el alumnado y sus familias, forma parte de los objetivos del presente artículo. Para ello se parte de un caso concreto, el actual CPI de Viaño Pequeno, situado en el municipio de Trazo. El relato de cómo se implantó este centro a través de la voz de una profesora que entonces formaba parte del cuadro docente, la información y los datos que proporciona, permiten hacer la crónica de una realidad que guarda muchas similitudes con la de los otros 53 centros públicos integrados distribuidos actualmente en la geografía de Galicia.

Para dar cuenta de todo ello se realiza inicialmente una breve descripción geográfica y se presentan algunas notas que ayudan a situar el caso de este centro en el entorno concreto donde está ubicado y, al mismo tiempo, se señalan algunos acontecimientos sobre cómo se fue fraguando el proceso de implantación de un centro público integrado en un municipio rural gallego.

Palabras clave: Centros Públicos Integrados, Educación Primaria, Educación Secundaria Obligatoria.

\begin{abstract}
The Organic Law 1/1990 provides for the operation of integrated centers covering two or more levels of education and the Royal Decree 1004/1991, June 14, in its first additional provision considers the operation of centers of different teachings in the same building or school site, determining the spaces
\end{abstract}


that are common and that allowing more flexible organizational forms to adapt to the different situations that the school reality may present. In this context the Department of Education and University Planning implements and regulates the existence in the educational organization of the Autonomous Community of Galicia of the integrated public centers (Decree 7/1999, of January 7, DOG núm.16, Tuesday, January 26 of 1999).

Reflecting on these issues, the opportunities that arised from the implementation of integrated public centers for students and their families is part of the objectives of this article. For this, we start from a specific case, the current CPI of Viaño Pequeno located in the municipality of Trazo. The story of how this center was implemented through the voice of a teacher who was then part of the teaching staff, the information and the data it provides, allow us to chronicle a reality that bears many similarities with that of the other 53 centers integrated publics currently distributed in the geography of Galicia.

To give an account of all this, a brief geographical description is initially made and some notes are presented that help to situate the case of this center in the specific environment where it is located and, at the same time, some events that show how it is located are indicated and was forging the process of implementing an integrated public center in a Galician rural municipality.

Keywords: Integrated Public Centers, Primary Education, Compulsory Secondary Education.

\section{INTRODUCCIÓN}

La Ley Orgánica 1/1990 prevé el funcionamiento de centros integrados que abarcan dos o más niveles de enseñanza. Por su parte, el Real Decreto 1004/1991, de 14 de junio, en su disposición adicional primera considera el funcionamiento de centros de diferentes enseñanzas en el mismo edificio o recinto escolar, determinando los espacios que son comunes y posibilitando formas organizativas más flexibles para adaptarse a las distintas situaciones que la realidad escolar puede presentar.

En este marco, la Consellería de Educación e Ordenación Universitaria implanta y regula la existencia en la organización educativa de la Comunidad Autónoma de Galicia de centros públicos integrados (Decreto 7/1999, de 7 de enero, DOG núm.16, Martes 26 de enero de 1999) con los que se pretende atender la demanda social existente, en especial en las zonas rurales, de modo que algunos alumnos y alumnas puedan realizar la educación obligatoria, ampliada a diez años, sin necesidad de cambiar de centro, aprovechando así los recursos existentes.

Según señala el artículo $2^{\circ}$ de este Decreto, en los Centros Públicos Integrados (CPI) se podrá impartir la educación básica en su totalidad y, además, es también posible impartir la educación infantil e incluso la educación de personas adultas o una combinación de enseñanzas de régimen general si así lo determina la Administración educativa; también se podrán impartir otras enseñanzas de régimen general no universitario.

La actividad docente de estos centros se ha de organizar y gestionar conjuntamente entre las distintas etapas educativas. En este sentido, es conveniente que el recinto escolar y las distintas instalaciones estén próximas o en un mismo edificio (artículo $3^{\circ}$ ), pudiendo las entidades locales contribuir al mantenimiento de los edificios escolares mediante la firma de convenios con la Administración educativa (Disposición adicional segunda). 
Asimismo, el Decreto 7/1999, de 7 de enero, por el que se implantan y regulan los centros públicos integrados de enseñanzas no universitarias (DOG, núm. 16, martes 26 de enero de 1999), señala en la Disposición adicional primera que se podrán constituir como centros públicos integrados que impartan educación secundaria obligatoria, aquellos colegios públicos de educación primaria o de educación infantil y primaria que atiendan poblaciones de especiales características sociodemográficas o escolares.

Respecto de la organización y gobierno de estos centros, el citado Decreto, en su Título I, Capítulo I, Artículo $1^{\circ} .6$, establece que el número de unidades de los CPI comprenderá todas las de educación básica y, cuando sea el caso, las de educación infantil y las correspondientes a otras enseñanzas impartidas en el centro.

Por lo que se refiere a su reglamento orgánico, cabe señalar, en primer lugar, que los órganos de gobierno de estos centros serán los previstos en el título II de la Ley Orgánica 9/1995, de 20 de noviembre, de participación, evaluación y gobierno de los centros docentes (Capítulo II, artículo $2^{\circ}$. 1); concretamente, los CPI deberán contar con los siguientes órganos unipersonales: director/a, jefe/a de estudios de educación infantil y primaria, jefe/a de estudios de educación secundaria y secretario/a o, de ser el caso, administrador/a; también dispondrán, como órganos de gobierno colegiados, de Consejo Escolar y de Claustro de Profesores (Título I, Capítulo II, artículo $3^{\circ}$ ).

En segundo lugar, en relación a los órganos de coordinación docente (Título II), los centros públicos integrados contarán con Equipos de ciclo en la educación primaria, Departamentos didácticos en la educación secundaria, Comisión de coordinación pedagógica, Tutores/as, Equipo de normalización lingüística, Departamento de orientación, Equipo de actividades extraescolares y complementarias, atención a los alumnos/as con necesidades educativas especiales $\mathrm{y}$, cuando se impartan dos o más grupos de ciclos formativos o programas de garantía social, Coordinación de formación en centros de trabajo (Capítulo I, artículo $13^{\circ}$ ).

En el día a día de un centro educativo, un aspecto fundamental es el referido a la participación de la comunidad educativa en la vida escolar. En este sentido, cabe señalar en relación a los CPI, lo recogido en el Título $\mathrm{V}$, artículo $85^{\circ}$, donde se indica que:

En los centros públicos integrados podrán existir, de acuerdo con la legislación vigente, asociaciones de padres y madres de alumnos y asociaciones de alumnos y alumnas, así como federaciones y confederaciones de ellas. En los reglamentos de régimen interior se garantizará, por parte del centro, los espacios pertinentes para la realización de actividades permanentes.

Con el fin de fomentar la participación y el enriquecimiento de la vida escolar del centro con otras estructuras, los centros públicos integrados podrán constituir otros órganos de participación y colaboración.

Dentro del marco legislativo vigente, los CPI dispondrán de la autonomía necesaria para definir el modelo de gestión organizativa y pedagógica, que deberá concretarse mediante un proyecto educativo de centro, los proyectos curriculares y el reglamento de régimen interior (Título III, Capítulo I, artículo $56^{\circ}$ ). 
Conviene por último recordar que en la regulación legislativa de los CPI está previsto que, teniendo en cuenta las circunstancias en las que se desarrollan las actividades educativas y la dotación de recursos humanos y materiales de los centros públicos integrados, la Consellería de Educación e Ordenación Universitaria, a través de la Inspección educativa, realice periódicamente una evaluación externa, para lo que es necesario contar con la colaboración de los órganos de gobierno colegiados y unipersonales del centro, de los órganos de coordinación didáctica, de los distintos sectores de la comunidad educativa y del profesorado (Título VI, artículo $87^{\circ}$ ). Del mismo modo, los propios centros, con el impulso de los órganos de gobierno y de la coordinación pedagógica, evaluarán su propio funcionamiento, sin prejuicio de lo que disponga en este sentido el proyecto educativo y curricular del centro (Título VI, artículo 88º).

Reflexionar sobre estas cuestiones, las oportunidades que se derivan de la puesta en marcha de los centros públicos integrados para el alumnado y sus familias, forma parte de los objetivos del presente artículo en el que se intenta reflejar un momento no muy lejano en el tiempo, de trascendencia en la realidad escolar, en especial en las zonas rurales de Galicia, en el que se produjo la implantación de los centros públicos integrados. Para ello se parte de un caso concreto, el actual CPI de Viaño Pequeno situado en el municipio de Trazo.

El relato de cómo se implantó este CPI a través de la voz de una profesora que entonces formaba parte del cuadro docente, la información y los datos proporcionados a lo largo de la entrevista realizada, permiten hacer la crónica de una realidad que, aunque singular y única, con toda seguridad guarda muchas similitudes con la de los otros 53 centros públicos integrados distribuidos actualmente en la geografía gallega.

Para dar cuenta de todo ello se realiza inicialmente una breve descripción geográfica y se presentan algunas notas que ayudan a situar el caso de este centro en el entorno concreto donde está ubicado $\mathrm{y}$, al mismo tiempo, se señalan algunos acontecimientos que dan cuenta de cómo se fue fraguando el proceso de implantación de un centro público integrado en un municipio rural gallego.

\section{DESCRIPCIÓN GEOGRÁFICA Y SOCIOECONÓMICA DEL MUNICIPIO DE TRAZO}

En la provincia de A Coruña, Comarca de Ordes, se sitúa el Municipio de Trazo, que limita al norte con el Municipio de Tordoia, al sur con el de Santiago de Compostela, al este con los de Tordoia y Oroso y al oeste con el de Val do Dubra. La capital municipal corresponde a Viaño Pequeno, lugar de la parroquia de San Juan de Campo, donde se encuentra ubicado el CPI de cuya descripción se ocupa este trabajo.

El municipio de Trazo tiene una superficie total de 100,48 kilómetros cuadrados que se reparten entre un total de once parroquias: Benza, Berreo, Campo, Castelo, Chaián, Monzo, Morlán, Restande, Trazo, Vilouchada y Xavestre. Todas estas parroquias son ZPP (Zonas Poco Pobladas) de acuerdo con lo establecido en la Clasificación das parroquias segundo o grao e subgrao de urbanización por el IGE (Instituto Gallego de Estadística, 2016). 


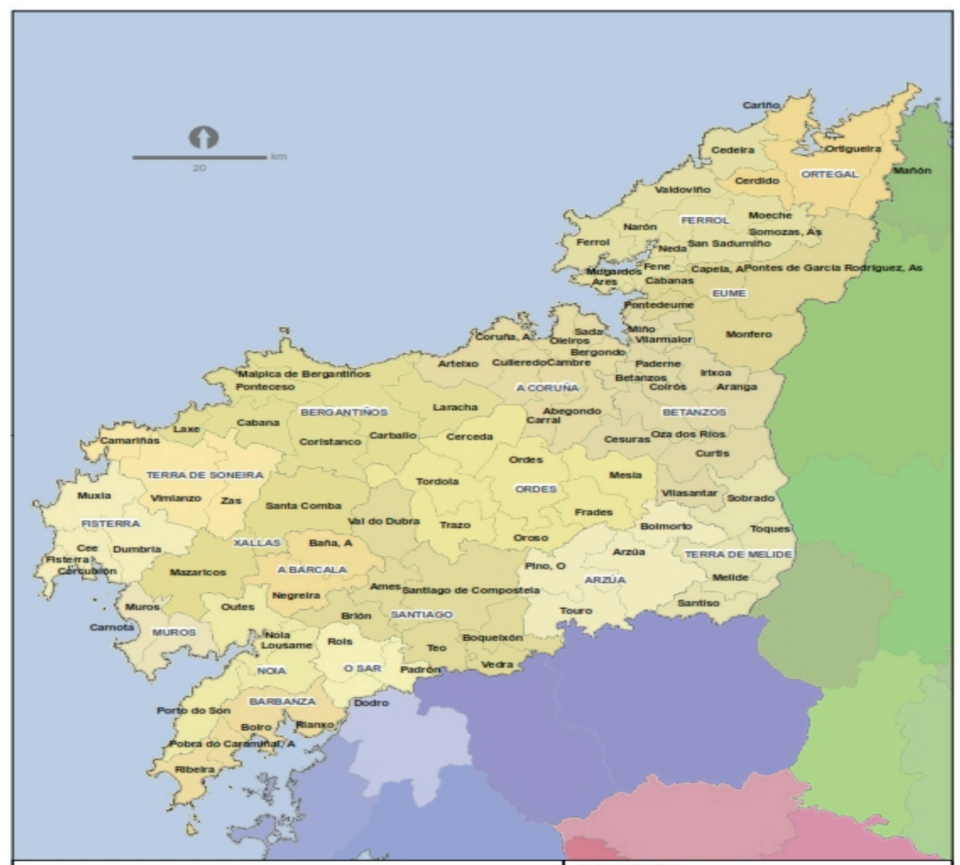

Figura 1. Situación del municipio de Trazo. Fuente: Cesga.

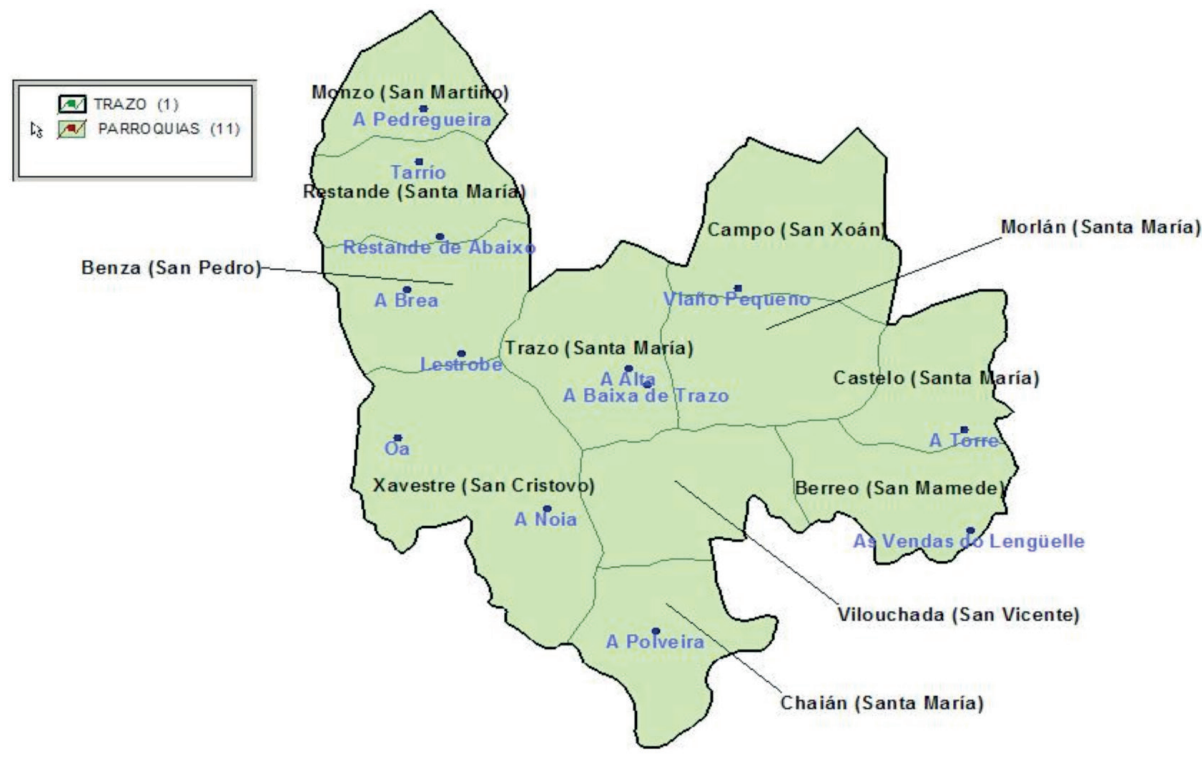

Figura 2. Situación de las parroquias del Ayuntamiento de Trazo. Fuente: Archivo Personal de Isabel Sánchez Manso. 


\begin{tabular}{lc}
\hline Parroquia & Superficie en $\mathbf{k m}^{\mathbf{2}}$ \\
\hline Benza (S. Pedro) & 9,5 \\
Berreo (S. Mamede) & 7,9 \\
Campo (S. Xoán) & 11 \\
Castelo (Sta. María) & 6,3 \\
Chaián (Sta María) & 7,9 \\
Monzo (S. Martiño) & 7,1 \\
Morlán (Sta. María) & 10,6 \\
Restande (Sta María) & 7,5 \\
Trazo (Sta María) & 9,5 \\
Vilouchada (S. Vicente) & 9 \\
Xavestre (S. Cristovo) & 17,1 \\
\hline
\end{tabular}

Tabla 1. Datos de superficie de las parroquias del municipio de Trazo. Fuente: IGE.

A comienzos del año 2000, cuando se implanta el CPI de Viaño Pequeno, la población con que contaba el municipio era de 3.818 habitantes.

Las cifras de población a 1 de enero de 2017 confirman un descenso y un envejecimiento poblacional al ser 3.186 el total de habitantes.

Respecto a los datos de escolarización del Municipio de Trazo, de acuerdo con la información recogida en el Mapa Escolar en el curso 1998-99, la población de 4 a 18 años, (según la revisión del Padrón de 1998) es de 675 sujetos, de ellos 68 es alumnado de Educación infantil, 259 de Educación primaria y 162 de la ESO.

\begin{tabular}{|c|c|c|}
\hline \multicolumn{3}{|c|}{ Total } \\
\hline Educación infantil & Educación primaria & Educación secundaria obligatoria (ESO) \\
\hline 68 & 259 & 162 \\
\hline
\end{tabular}

Tabla 2. Datos de escolarización en el Municipio de Trazo en el curso 1998-99. Fuente: IGE.

En el año 2017, el número total de alumnos/as es de 210; cursan Educación infantil 45, Educación primaria 103 y la ESO 62, con una ligera mayoría de alumnos frente a alumnas en el cómputo global, si bien, cuando se observan las cifras en cada etapa educativa se comprueba que hay más niños en edad infantil que niñas; en primaria son mayoría las niñas y en la ESO hay un mismo número de alumnos que de alumnas.

\begin{tabular}{|ccc|ccc|ccc|}
\hline & \multicolumn{1}{c}{ Total } & \multicolumn{1}{c}{ Alumnos } & \multicolumn{3}{c}{ Alumnas } \\
\hline $\begin{array}{c}\text { Educación } \\
\text { infantil }\end{array}$ & $\begin{array}{c}\text { Educación } \\
\text { primaria }\end{array}$ & $\begin{array}{c}\text { Educación } \\
\text { secundaria } \\
\text { obligatoria } \\
\text { (ESO) }\end{array}$ & $\begin{array}{c}\text { Educación } \\
\text { infantil }\end{array}$ & $\begin{array}{c}\text { Educación } \\
\text { primaria }\end{array}$ & $\begin{array}{c}\text { Educación } \\
\text { secundaria } \\
\text { obligatoria } \\
(\text { ESO) }\end{array}$ & $\begin{array}{c}\text { Educación } \\
\text { infantil }\end{array}$ & $\begin{array}{c}\text { Educación } \\
\text { primaria }\end{array}$ & $\begin{array}{c}\text { Educación } \\
\text { secundaria } \\
\text { obligatoria } \\
(\text { ESO) }\end{array}$ \\
\hline 45 & 103 & 62 & 29 & 49 & 31 & 16 & 54 & 31 \\
\hline
\end{tabular}

Tabla 3. Datos de escolarización en el Municipio de Trazo en el año 2017. Fuente: IGE. 
En este municipio y según las afiliaciones a la Seguridad Social, la mayor parte del trabajo lo genera el sector servicios, al igual que sucede en Galicia. Sin embargo, la particularidad de esta zona es el gran peso que aún tiene el sector agrario, al ocupar a casi una cuarta parte de la población activa $(23,2 \%)$, frente a la importancia mucho menor -en cuanto a ocupación- que tiene en Galicia con un $5,6 \%$.

\begin{tabular}{|c|c|c|c|c|}
\hline \multirow{2}{*}{ Sectores de actividad } & \multicolumn{2}{|c|}{ Municipio de Trazo } & \multicolumn{2}{|c|}{ Galicia } \\
\hline & v.a. & v.r. & v.a. & v.r. \\
\hline Agricultura & 305 & 23,2 & 58.660 & 5,8 \\
\hline Industria & 161 & 12,3 & 144.093 & 15,1 \\
\hline Construcción & 141 & 10,7 & 74.091 & 7,1 \\
\hline Servicios & 705 & 53,8 & 712.777 & 72,0 \\
\hline TOTAL & 1.312 & 100 & 989.621 & 100 \\
\hline
\end{tabular}

Tabla 4. Estructura sectorial. Filiaciones en alta laboral por sectores en el municipio de Trazo y Galicia Fuente. SS, IGE.

\section{ANTES DE LA IMPLANTACIÓN DEL CENTRO PÚBLICO INTEGRADO}

Hay que remontarse a los primeros años de la década de los setenta del pasado siglo para conocer los inicios y la creación del primer centro de enseñanza en el Municipio de Trazo, al que acude alumnado de las distintas parroquias de la zona.

En efecto, en enero de 1973, el Pleno municipal de Trazo acuerda poner a disposición de la "Junta Central de Construcciones, Instalaciones y Equipo Escolar" dos parcelas de una superficie de 15.777 metros cuadrados, ubicadas en el lugar de Viaño Pequeno, parroquia de Campo, terrenos cedidos por doce propietarios ante la Alcaldía y el representante del "Instituto de Reforma y Desarrollo Agrario (IRYDA), organismo que en su día deberá compensar a esos propietarios la cesión realizada.

El 27 de julio de 1974, la citada "Junta Central de Construcciones, Instalaciones y Equipo Escolar" del Ministerio de Educación y Cultura comunica al Ayuntamiento de Trazo la aceptación de las parcelas referidas en las que se construirá un Colegio Nacional.

En el Boletín Oficial del Estado del 14 de noviembre de 1975 se publica la Resolución de la "Junta de Construcción, Instalaciones y Equipo Escolar" del Ministerio de Educación y Ciencia en la que se anuncia el concurso-subasta de las obras de construcción de un centro de Educación General Básica de 8 unidades, por un importe de 18.996 .706 pesetas, cantidad que finalmente se reduce, adjudicándose definitivamente las obras por valor de 16.004.727 pesetas.

Aproximadamente un año más tarde, en el mes de marzo de 1976, el Pleno Municipal de Trazo solicita al entonces Ministerio de Educación y Ciencia la ampliación del centro escolar en seis unidades más para poder impartir en igualdad de condiciones la Educación General Básica a todo el alumnado del municipio. 
Con posterioridad, en mayo de 1978, la Inspección Provincial de Enseñanza Primaria comunica la subasta de las obras para la ampliación de 8 unidades en el denominado Colegio Nacional de Trazo que en ese momento contaba con 320 puestos escolares, obras que finalizarán un año después, de manera que el 21 de junio del año 1979 comienza el funcionamiento del edifício escolar destinado a albergar al alumnado de la Segunda Etapa de la Educación General Básica, esperando el transporte escolar para el alumnado de la primera etapa.

En el último trimestre del año 1980, la Delegación Provincial de Educación comunica al Ayuntamiento la desafectación de los locales en los que funcionaban las escuelas que ahora se concentrarán en el Colegio Nacional de Trazo (Orden Ministerial de 4 de diciembre de 1979): Local de la Escuela mixta de Berreo-Vilasuso; Local de la Escuela de niñas de Campo; Local de la Escuela de niños de Campo; Local de la Escuela mixta de Castelo; Local de la Escuela de niños de Chaián; Local de la Escuela mixta de Monzo; Local de la Escuela de niños de Morlán; Local de la Escuela de niñas de Morlán; Local de la Escuela de niños de Restande; Local de la Escuela de Xavestre.

Finalmente, el 23 de septiembre y el 12 de diciembre de 1980 tiene lugar la recepción definitiva de la nueva construcción y reforma adicional a la nueva construcción de un Centro de Educación General Básica de 8 unidades y la correspondiente a la construcción y adaptación de las unidades de Educación Preescolar respectivamente.

El centro quedará pues conformado en este momento por dos edificios; uno de ellos ocupado por los/as alumnos/as de Educación Infantil y de Primer Ciclo de Educación General Básica, y otro, el edificio central, en el que se sitúan instalaciones comunes como el comedor, la cocina, las dependencias administrativas y las aulas destinadas al alumnado del Segundo y Tercer Ciclo de Educación General Básica.

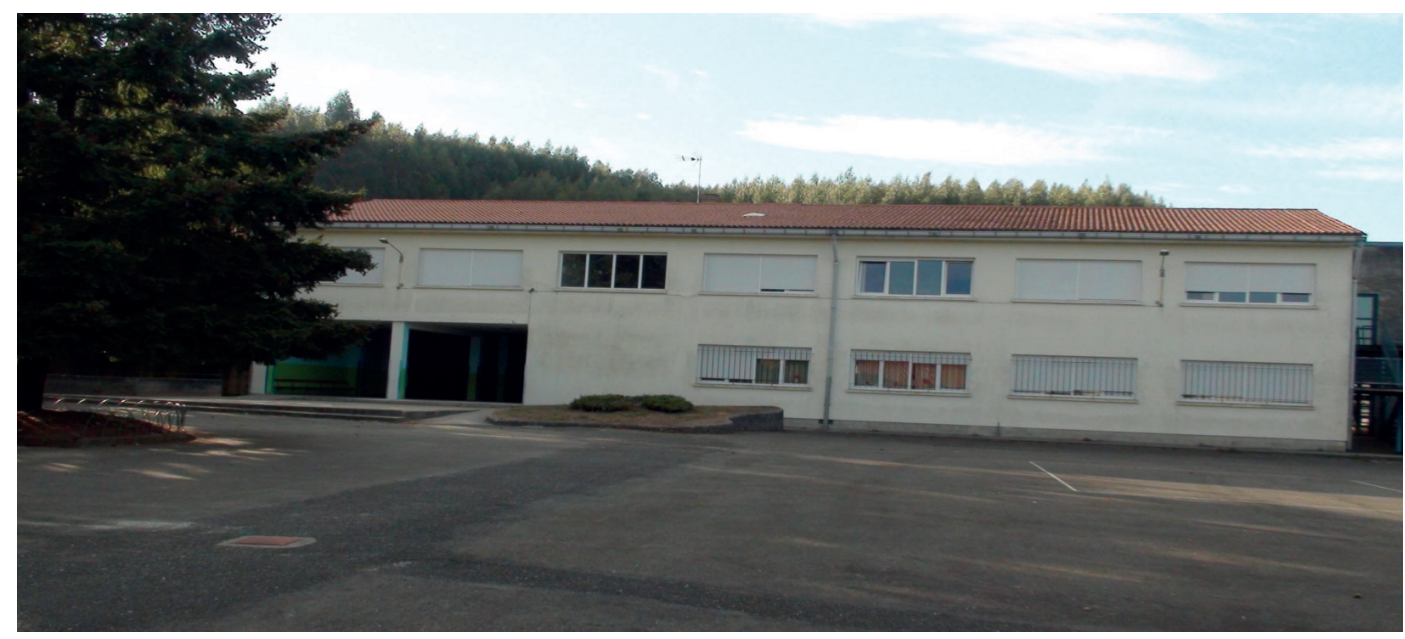

Figura 3. Edificio Educación Infantil CPI Viaño Pequeno. Fuente: Dirección Centro CPI Viaño Pequeño. 


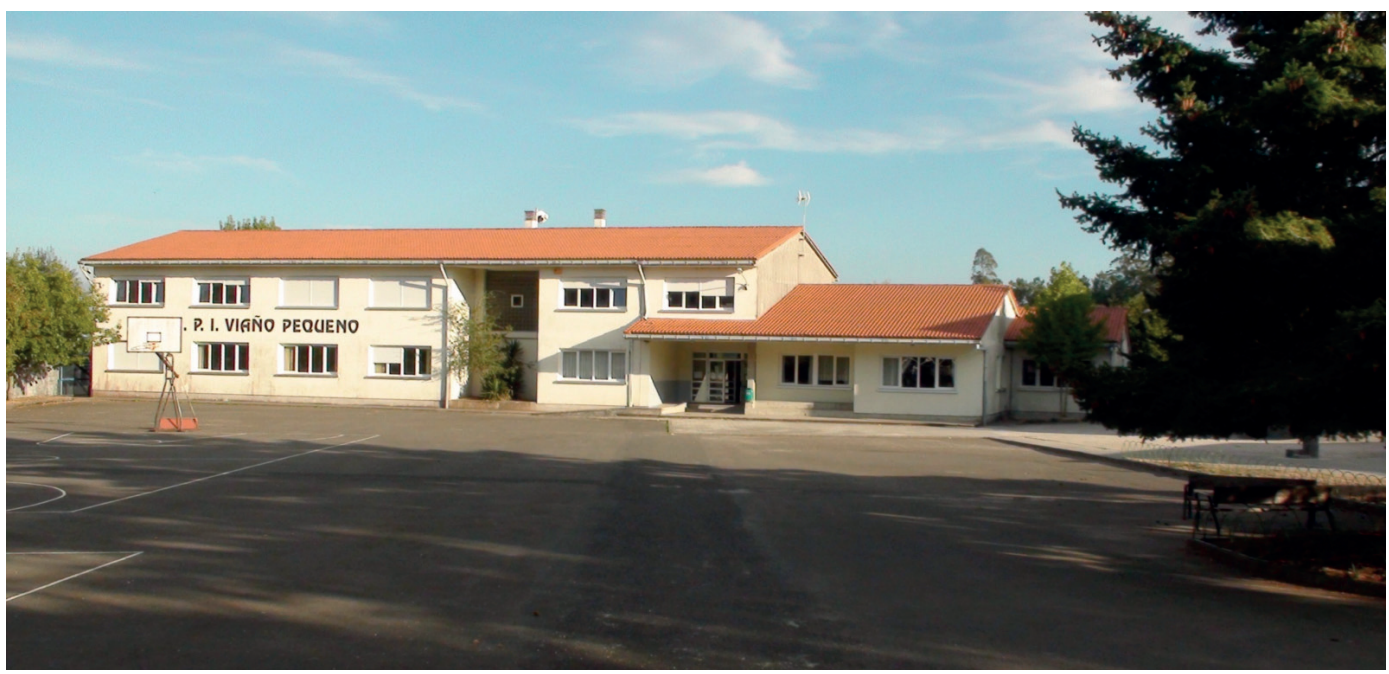

Figura 4. Edificio Central CPI Viaño Pequeno. Fuente: Dirección Centro CPI Viaño Pequeño.

\section{LA IMPLANTACIÓN DE LA ESO EN EL CPI DE VIAÑO PEQUENO}

De acuerdo con Ley Orgánica 1/1990, de 3 de octubre, de Ordenación General del Sistema Educativo, (BOE» núm. 238, de 4 de octubre de 1990), Título Preliminar, artículo 3.2. las enseñanzas de régimen general se ordenan en: Educación infantil, Educación primaria, Educación secundaria, que comprenderá la educación secundaria obligatoria, el bachillerato y la formación profesional de grado medio, Formación profesional de grado superior y Educación universitaria. La educación primaria y la educación secundaria obligatoria constituyen la enseñanza básica. La enseñanza básica comprenderá diez años de escolaridad, iniciándose a los seis años de edad y extendiéndose hasta los dieciséis (artículo 5.1) y será obligatoria y gratuita (artículo 5.2).

La educación infantil comprenderá dos ciclos. El primer ciclo se extenderá hasta los tres años y el segundo desde los tres hasta los seis años de edad (Titulo Primer de las Enseñanzas de Régimen General. Capítulo Primero, De la educación infantil, Art. 9). La educación primaria comprenderá seis cursos académicos, desde los seis a los doce años de edad (Capitulo II. De la Educación Primaria, Artículo 12) y tres ciclos de dos cursos académicos cada uno y se organizará en áreas que serán obligatorias y tendrán un carácter global e integrado. La finalidad de este nivel educativo será proporcionar a todos los niños una educación común que haga posible la adquisición de los elementos básicos culturales, los aprendizajes relativos a la expresión oral, a la lectura, a la escritura y al cálculo aritmético, así como una progresiva autonomía de acción en su medio (Capitulo II. De la educación primaria, Artículo 14).

El nivel de educación secundaria comprenderá, junto al bachillerato y la formación profesional, la etapa de educación secundaria obligatoria, que completa la enseñanza básica y abarca cuatro cursos académicos, entre los doce y dieciséis años de edad (Capítulo II De la educación primaria, Artículo 12, 
a). Esta etapa constará de dos ciclos, de dos cursos cada uno, y se impartirá por áreas de conocimiento (Capítulo III De la educación secundaria, Artículo 20.1). Cabe señalar que impartirán las enseñanzas de esta etapa licenciados/as, ingenieros/as y arquitectos/as o quienes posean una titulación equivalente a efectos de docencia (Capítulo III De la educación secundaria, Artículo 24.1). Además, será necesario estar en posesión de un título profesional de especialización didáctica. Este título se obtendrá mediante la realización de un curso de cualificación pedagógica, con una duración mínima de un año académico, que incluirá, en todo caso, un período de prácticas docentes. El Gobierno regulará las condiciones de acceso a este curso y el carácter y efectos de los correspondientes títulos profesionales, así como las condiciones para su obtención, expedición y homologación. Las Administraciones educativas podrán establecer los correspondientes convenios con las universidades al objeto de la realización del mencionado curso (Capítulo III De la educación secundaria, Artículo 24.2).

Finalmente, cabe señalar que la Ley, en su Titulo V, De la Compensación de las Desigualdades en la Educación señala en el Artículo 65.1: En el nivel de educación primaria, los Poderes públicos garantizarán a todos los alumnos un puesto escolar gratuito en su propio municipio en los términos que resultan de la aplicación de la Ley Orgánica del Derecho a la Educación.

En el mismo Artículo 65.2 se indica que: Excepcionalmente, en la educación primaria y en la educación secundaria obligatoria en aquellas zonas rurales en que se considere aconsejable, se podrá escolarizar a los niños en un municipio próximo al de su residencia para garantizar la calidad de la enseñanza. En este supuesto las Administraciones educativas prestarán de forma gratuita los servicios escolares de transporte, comedor y, en su caso, internado.

En el año académico 1999-2000 se implanta el CPI de Viaño Pequeno en el Ayuntamiento de Trazo, momento en el que se inaugura un tercer edificio, el de más reciente construcción, que acogerá al alumnado de la ESO.

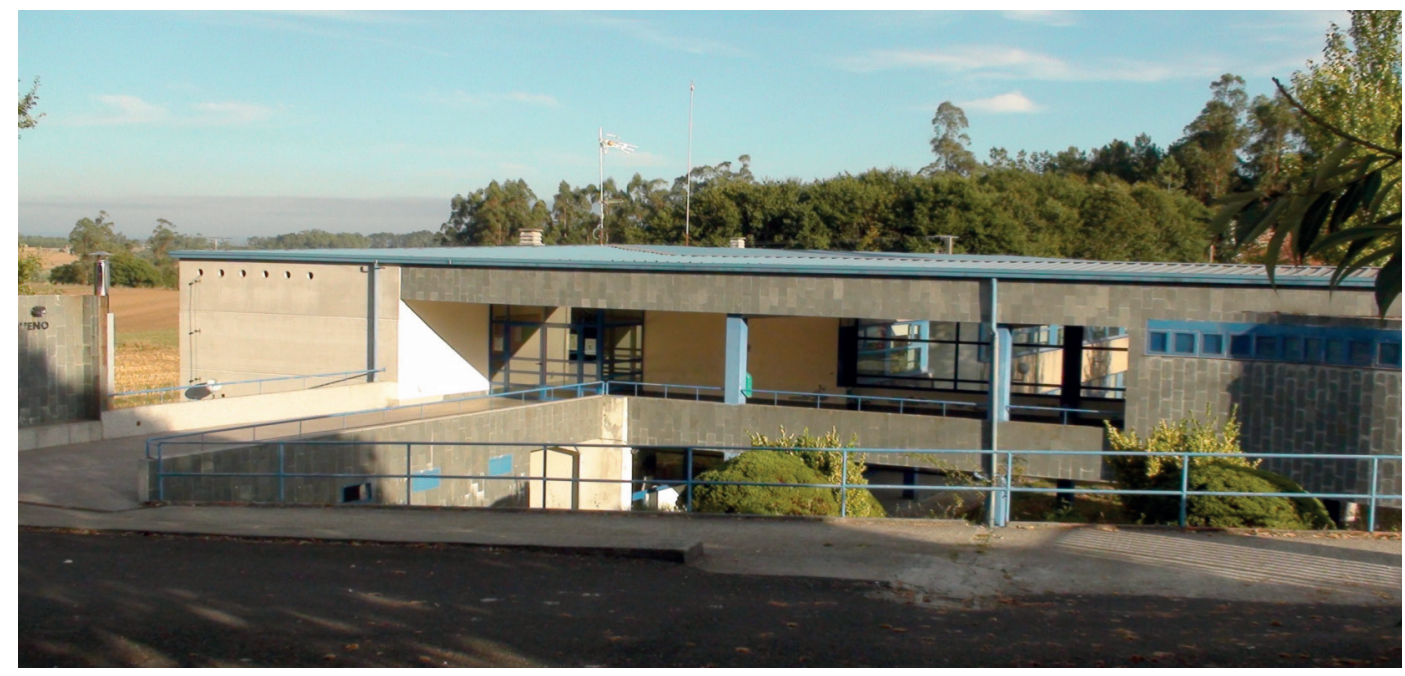

Figura 5. Edificio ESO CPI Viaño Pequeno. Fuente: Dirección Centro CPI Viaño Pequeño. 
En la nueva configuración educativa de este centro se impartirán estudios de educación infantil, de educación primaria con sus tres ciclos inicial, medio y superior y de educación secundaria obligatoria, que en ese curso contará con alumnos y alumnas distribuidos/as en cinco aulas en el Primer Ciclo y cuatro en el Segundo Ciclo.

Hay que tener en cuenta que esta nueva situación supuso para el centro importantes cambios organizativos y también implicó el desarrollo de adaptaciones para lograr el éxito y una acertada puesta en marcha de la nueva etapa educativa.

Las principales acciones que se tuvieron que afrontar en el centro, tal y como revela en una entrevista Isabel, docente con destino definitivo desde el año 1986 hasta el final de su actividad educativa en 2015 en el CPI de Viaño Pequeño, fueron principalmente:

— "Incremento de la plantilla de profesorado";

— "Reparto de los espacios para actividades deportivas, utilizando los cedidos por la Administración local, mientras no se disponía de un pabellón propio";

— "Distribución de los espacios para llevar a cabo las actividades académicas";

— "Ubicación del alumnado de cada curso en las aulas más adecuada";

— "Elaboración de nuevos horarios, ajustados a las distintas necesidades de los diferentes cursos y de cada etapa educativa".

La formación y preparación del profesorado del centro para este nuevo reto fue fundamental, según indica esta docente. En este sentido señala que se realizaron diferentes actividades formativas, entre otras:

— "Cursos para la utilización de los programas XADE y CLIC";

— "Cursos sobre el uso didáctico de las nuevas tecnologías";

_ "Curso para la elaboración de materiales de apoyo".

Fue también necesario compartir espacios. Concretamente, la nueva estructura implicó:

— "Compartir lugares para la realización das distintas actividades";

— "Compartir el polideportivo, siguiendo una adecuada y compatible distribución horaria";

— "Utilizar espacios complementarios, fuera del horario lectivo, por ejemplo, el aula de informática o la biblioteca escolar".

Pese a todo, las oportunidades que esta situación proporcionó a toda la comunidad educativa de este centro no pueden ser olvidadas, por el contrario, deben ser consideradas como valiosas, especialmente pensando en el alumnado y sus familias. En este sentido, la entrevistada valora positivamente: 
- La ubicación del alumnado en su municipio, más proximidad a su aldea o parroquia, en definitiva, proximidad a su casa;

- La socialización del alumnado de las distintas parroquias dos años más, utilizando el transporte escolar y conviviendo en esta etapa en el centro;

- Mayor contacto y proximidad de las familias con el centro;

Para el centro y el profesorado señala que se generaron nuevas oportunidades, por ejemplo:

- "Mayor contacto con las familias, en una etapa evolutiva tan importante y de cambios para el alumnado";

— "Oportunidad para que el alumnado pueda participar en algunos proyectos con compañeros/as de distintas edades y niveles";

— "Despertar en el alumnado el sentido de pertenencia a su entorno y al centro";

\section{A MODO DE REFLEXIÓN FINAL}

Los centros públicos integrados regulados en Galicia a través del Decreto 7/1999, de 7 de enero (DOG núm. 16, Martes 26 de enero de 1999) son una respuesta para que en las zonas rurales alumnos y alumnas puedan cursar la educación obligatoria, ampliada a diez años, sin necesidad de cambiar de centro, aprovechando así los recursos existentes; un formato que parece pensado, sobre todo “.... para evitar los largos desplazamientos de los alumnos y el desarraigo prematuro de su localidad” (Rubia, 2011, p. 33).

Los CPI permiten la transición del alumnado de una etapa educativa a otra de una forma más normalizada, permaneciendo en su centro de origen durante más tiempo. Prolongar unos años más la escolarización en un centro conocido hace que sea más fácil para el alumnado relacionarse con sus compañeros/as y conocerse mejor, dando lugar a más oportunidades de socialización, al tiempo que se desarrolla el sentimiento de pertenencia.

Como señala Rubia (2011, p. 37):

El actual sistema público fuerza al cambio de centro al acabar la primaria, precisamente en una edad considerada socialmente inmadura y en la que los niños pueden sufrir la pérdida de sus amistades si adoptan decisiones independientes. Esto puede tener como consecuencia un primer inconveniente, la desestabilización emocional.

La posibilidad de continuar en el mismo centro toda la educación obligatoria es para muchos alumnos y alumnas, y también para sus familias, especialmente de los núcleos rurales, una buena opción para afrontar esa etapa educativa.

Con todo, parece necesario acompañar la creación de esta estructura educativa con el apoyo y desarrollo económico del entorno, esto es, con medidas socio económicas para el desarrollo de una estructura que pueda dar a los municipios rurales más peso económico y ayude a fijar población, crear nuevas perspectivas de vida o buscar novas formas para la explotación de los recursos existentes. 
La información obtenida sobre la realidad de un CPI concreto, fruto de la entrevista realizada a una docente que ejerció su actividad durante más de treinta años en el mismo permite, entre otras cosas, conocer de primera mano algunos de los cambios que ha experimentado la escuela en época reciente. El centro público integrado referido y los otros que como él fueron implantados hace casi dos décadas hacen posible que niños y niñas de los municipios rurales de Galicia permanezcan unos años más de la escolaridad obligatoria en su entorno, sin tener que romper a una edad temprana con sus primeras vivencias y trasladarse a otra localidad para realizar sus estudios.

Quizá merezca la pena que se dedique algún tiempo y esfuerzo a estudiar con detenimiento las posibilidades que esta opción puede proporcionar.

\section{REFERENCIAS BIBLIOGRÁFICAS}

CESGA (s. f.). Servidor de mapas. Recuperado de http://archivo.cesga.es/content/view/498/84/ lang,es/

Decreto 7/1999, de 7 de enero, por el que se implantan y regulan los centros públicos integrados de enseñanzas no universitarias. Diario Oficial de Galicia, 26 de enero de 1999, núm. 16, p. 877-906.

Ley Orgánica 1/1990, de 3 de octubre, de Ordenación General del Sistema Educativo, Boletín Oficial del Estado, 4 de octubre de 1990, núm. 238, p. 28927-28942.

Instituto Gallego de Estadística (s. f.). Datos del padrón municipal de habitantes. https://www.ige. eu/web/mostrar_actividade_estatistica.jsp?idioma $=$ es\&codigo $=0201001002$

Instituto Gallego de Estadística (2016). Clasificación das parroquias segundo o grao e subgrao de urbanización. http://www.ige.eu/web/mostrar_paxina.jsp?paxina=003003001\&idioma=es

Real Decreto 1004/1991, de 14 de junio, por el que se establecen los requisitos mínimos de los Centros docentes que impartan enseñanzas de régimen general no universitarias. Boletín Oficial del Estado, 26 de junio de 1991, núm. 152, p. 21181 a 21187

Rubia, F. A. (2011). Los centros públicos integrados de enseñanza obligatoria: un territorio inexplorado. Fórum Aragón: revista digital de FEAE-Aragón sobre organización y gestión educativa, 3, 33-37. 